\title{
How should radiation be done for inflammatory breast cancer patients? - a narrative review of modern literature
}

\author{
Kelsey L. Corrigan ${ }^{1}$, Wendy A. Woodward ${ }^{1,2}$, Michael C. Stauder ${ }^{1,2}$ \\ ${ }^{1}$ Department of Radiation Oncology, the University of Texas MD Anderson Cancer Center, Houston, TX, USA; ${ }^{2}$ The Morgan Welch IBC Clinic \\ and Research Program, UT MD Anderson Cancer Center Houston, TX, USA \\ Contributions: (I) Conception and design: MC Stauder; (II) Administrative support: WA Woodward; (III) Provision of study materials or patients: \\ WA Woodward, MC Stauder; (IV) Collection and assembly of data: All authors; (V) Data analysis and interpretation: MC Stauder; (VI) Manuscript \\ writing: All authors; (VII) Final approval of manuscript: All authors. \\ Correspondence to: Michael C. Stauder, MD. Division of Radiation Oncology, University of Texas MD Anderson Cancer Center, 1515 Holcombe Blvd, \\ Unit 1202, Houston, TX, USA. Email: mstauder@mdanderson.org.
}

Objective: This review highlights the considerations of the radiation oncologist when managing patients with inflammatory breast cancer (IBC) as well as the radiation oncologist's role as a member of the multidisciplinary team.

Background: IBC makes up only $1-4 \%$ of all breast cancer diagnoses but incidence is increasing. IBC is diagnosed based on a constellation of clinical features, including the rapid onset of breast erythema and edema (peau d'orange) of one-third or more of the skin of the breast and with a palpable border to the edema. Most published IBC local-regional control rates are consistently lower than those observed in nonIBC, which the highlights the need for deliberate treatment techniques to maximize clinical outcomes.

Methods: For this narrative review, we discuss the principles of radiation target delineation and dose escalation; highlight new findings in the local-regional management of IBC; provide a critical evaluation of the recent literature evaluating local-regional treatment of IBC; and offer a brief introduction to possible future directions regarding the optimal treatment and management of IBC based on our institutional experience.

Conclusions: IBC is an aggressive type of breast cancer that warrants multi-disciplinary care from breast surgical, medical, and radiation oncology. Several strategies exist to enhance the effect of radiation therapy (RT) on local-regional control, including hyperfractionation, use of bolus, increased total RT dose, and radiosensitizers, which are currently being tested in randomized trials. With an individualized patient approach, local-regional control rates are improving for IBC.

Keywords: Inflammatory breast cancer; post-mastectomy; radiation therapy; breast cancer

Submitted Nov 11, 2021. Accepted for publication Dec 17, 2021.

doi: 10.21037/cco-21-153

View this article at: https://dx.doi.org/10.21037/cco-21-153

\section{Introduction}

The diagnosis of inflammatory breast cancer (IBC) comprises a small subset of all breast cancers in the United States yet accounts for up to $10 \%$ of breast cancer mortality. Key clinical outcome measures, such as overall survival (OS), are significantly worse for IBC compared to non-IBC (1). Additionally, the incidence of IBC is increasing. According to the Surveillance, Epidemiology and End Results Program
(SEER) and North American Association of Central Cancer Registries, the incidence of IBC has more than doubled over the past 30 years, an increase which is greater than that of non-IBC (2). Despite the increased incidence, OS has changed only slightly over the same period. This has been primarily attributable to advances in systemic chemotherapy since local-regional control (LRC) rates have remained relatively constant (3). 
The increasing IBC incidence without improvement in OS highlights the need for deliberate treatment techniques to maximize clinical outcomes for patients with IBC. In this narrative review, we aim to describe modern treatment techniques based on our institution's experience as well as the literature over the past 20 years. We discuss the principles of radiation target delineation and dose escalation; highlight new findings in the local-regional management of IBC; provide a critical evaluation of the recent literature evaluating local-regional treatment of IBC; and offer a brief introduction to possible future directions regarding the optimal treatment and management of IBC. We present the following article in accordance with the Narrative Review reporting checklist (available at https:// dx.doi.org/10.21037/cco-21-153).

\section{Multi-disciplinary approach to IBC aids radiation oncology decisions}

As part of the multi-disciplinary approach to IBC in a highvolume breast cancer specialty clinic, it is value added to prioritize the involvement of breast surgical, medical, and radiation oncology at the time of diagnosis. Documentation of the skin involvement using medical photography and careful documentation of the extent of disease on physical exam prior to neoadjuvant chemotherapy is critical to determine the extent of upfront skin involvement. Specifically, it is important for the radiation oncologist to visually inspect the extent of skin disease, including any extension beyond the breast mound at the time of diagnosis, as it will often guide surgical and radiation planning to ensure that all erythema has adequate RT field and dose coverage.

The use of cross-sectional imaging, such as PET/CT and MRI, at diagnosis is also helpful in establishing eventual radiation therapy (RT) field borders and ensuring adequate dose coverage to initially involved nodal areas (4). In IBC patients, PET-CT, MRI, or CT of the chest and neck often demonstrate involved level III and supraclavicular nodes that are deep compared to standard breast cancer RT dose distributions. In addition, diagnostic imaging reports often do not adequately convey all information relevant to RT planning. Thus, personal review of these pre-chemotherapy films is critical. All patients with $\mathrm{N} 3 \mathrm{c}$ disease noted on chest CT should have a cross-sectional imaging through the neck (neck CT or PET/CT) to localize all pre-chemotherapy disease after surgery.

While uncommon in non-IBC patients, involvement of contralateral lymph node basins is more likely in IBC due to the aggressive nature of the disease and extensive remodeling of the lymphatics, which merits bilateral nodal imaging in these patients even when the contralateral breast is negative by imaging.

\section{Tri-modality therapy including radiotherapy improves outcomes}

In a National Cancer Data Base (NCDB) study of nonmetastatic patients with IBC, the 10 -year survival rate was highest for patients who received tri-modality therapy $(37.2 \%)$ compared to surgery alone $(16.5 \%)$ or dual modality therapy with surgery/chemotherapy $(28.5 \%)$ or surgery/radiation (23.5\%) (5). As a result, one may conclude that RT as a component of tri-modality care plays a key role in optimizing oncologic outcomes. Therefore, maximizing and personalizing the use of aggressive local therapy, which may include accelerated RT dose delivery (called hyperfractionation), use of bolus (tissue equivalent material to increase RT skin dose), and/or total RT dose escalation, are likely needed to improve local-regional control (LRC) rates in this population (6-8). Retrospective data examining non-IBC breast cancer patients treated with a contemporary regimen of neoadjuvant chemotherapy, modified radical mastectomy, and adjuvant RT demonstrated a 5-year LRC rate of $97 \%$, much higher than what is observed in IBC patients (9). According to a recent review examining single institution experiences, similarly staged IBC patient populations had 5 -year LRC rates ranging from $73 \%$ to $92 \%$ (10). These studies prompt the use of more aggressive RT or systemic therapy regimens. For example, there is a currently accruing randomized trial among IBC patients comparing standard dose and fractionation postmastectomy RT plus or minus a PARP inhibitor based on pre-clinical work suggesting radiosensitivity in IBC.

Although the total number of patients is small, some evidence has recently been presented suggesting that the use of hyperthermia in IBC may be beneficial. A Russian Federation study, presented in abstract form only, evaluating 14 IBC patients, combining chemotherapy with hyperthermia resulted in an $86 \%$ objective response rate (11). Only 1 local recurrence was seen in this group of patients with median OS of 50 months and median metastasis-free survival of 30 months.

\section{Aggressive radiation therapy regimens}

A large retrospective study from the University of Texas MD 
Table 1 Five- and 10-year rates of DFS and OS in patients with inflammatory breast cancer treated with conventional radiation therapy [60 Gy delivered once-daily (qd)] vs. dose-escalated radiation therapy [66 Gy delivered twice-daily (bid)]

\begin{tabular}{lcccc}
\hline Dose/fractionation & 5-year DFS (\%) & 10-year DFS (\%) & 5-year OS (\%) & 10-year OS (\%) \\
\hline 60 Gy qd & 32 & 28 & 40 & 33 \\
66 Gy bid & 38.7 & 34.7 & 46.3 & 36.5 \\
\hline
\end{tabular}

Adapted from: Liao et al. (13). DFS, disease-free survival; OS, overall survival.

Table 2 Relationship between dose and LRC in IBC

\begin{tabular}{|c|c|c|c|c|}
\hline Center & Dose & 5-year LRC & Era & Notes \\
\hline Cleveland & $\geq 60.4$ Gy & $100 \%$ & & \\
\hline Mayo & 60-66 Gy, qd bolus & $81 \%$ & 2000-2010, N=49 & Pathologic complete response associated with better LRC \\
\hline Penn & 46-60 Gy, qd bolus & $88 \%$ & $1986-2006, N=19^{b}$ & Only patients with dermal lymphatic invasion had LRR \\
\hline BCCA & 42.4 Gy, bolus NS ${ }^{d}$ & $63 \%$ & $1980-2000, N=148$ & Pathologic complete response associated with better LRC \\
\hline
\end{tabular}

Adapted from: Woodward et al. (15). ${ }^{a}$, LRR for patients with negative margins; ${ }^{\mathrm{b}}$, LRR for IBC patients with dermal lymphatic invasion; ${ }^{\mathrm{c}}$, 11 patients received a boost to $60 \mathrm{~Gy}, 11$ did not complete the prescribed treatment; ${ }^{\mathrm{d}}, 80 \%$ received this dose. $20 \%$ received standard fractionation. prn bolus, bolus as needed; bolus NS, bolus not specified; bid, treatment twice daily; MVA, multivariable analysis; LRR, local-regional recurrence; IBC, inflammatory breast cancer; LRC, local-regional control.

Anderson Cancer Center showed increased LRC compared to historical standards using utilizing hyperfractionated and dose-escalated RT regimens (12). Specifically, the routine use of dose-escalated RT to 66 Gy delivered twice-daily (BID) for the treatment of IBC improved rates of diseasefree survival and OS (Table 1). Age younger than 45 years old, incomplete response to chemotherapy (either residual breast or nodal disease), and inadequate surgical margins were identified as high-risk factors for local recurrence in this study and, moving forward after this publication, were used to de-escalate radiation in IBC patients without these features (6). The twice daily regimen consists of 51 Gy to the chest wall and draining lymphatic regions, including the axillary levels I-III, supraclavicular, and internal mammary nodes, and a sequential 15 Gy boost to all areas of skin involved at diagnosis and to areas with evidence of surgical change. The entire treatment is given at a dose of 1.5 Gy per fraction, delivered BID. A more recent report of outcomes after de-escalation of RT reserving the BID regimen for patients under 45 years old or with residual disease after chemotherapy was associated with outstanding 3-year local control: 95\% among the higher risk BID patients and $100 \%$ among the patients treated with the daily RT regimen (14).

These institutional studies demonstrating improved LRC with RT hyperfractionation, bolus, or RT dose escalation are corroborated by other published contemporary literature. Investigators from Cleveland Clinic report a $100 \%$ 5 -year LRC rate in patients receiving more than 60.4 Gy compared to $83 \%$ in those receiving 60.4 Gy or less (8). A more recent study from Mayo Clinic, which details their institutional experience with once-daily RT (qd), has comparable results, reporting 5 -year LRC of $81 \%$. In this experience, the aggressive use of daily skin bolus substituted for an increased total RT dose, however it resulted in a $46 \%$ rate of grade 3 radiation dermatitis (15). In another study of 107 patients treated with a moderate RT dose (median $50.4 \mathrm{~Gy}$ ) along with daily skin bolus, the 5 -year LRC rate was $87 \%$. However, patients who received a total RT dose of $>60$ Gy had a $100 \%$ LRC at 5 years (7). Among these recent studies (Table 2), RT hyperfractionation, bolus, and/or increased total RT dose play important roles in local control and 
that one of these strategies is warranted in all cases. In conclusion, IBC is an aggressive disease and therefore requires aggressive $\mathrm{RT}$ regimens involving the described strategies (16).

\section{Treatment volumes}

Skin involvement is the hallmark of IBC; thus, it is an important therapeutic target. As previously mentioned, medical photographs and diagnostic images prior to beginning systemic therapy are critical in establishing the extent of disease at the outset, which should be used to guide RT target delineation. An adequate, homogeneous skin RT dose to all affected areas and margin is needed for successful outcomes. Furthermore, radiation oncologists should plan and match 3-D conformal RT fields such that no gaps exist on the skin. The overlapping of 3-D conformal field borders by $2-3 \mathrm{~mm}$ in areas where the skin was involved is one approach to avoid this issue and is generally associated with minimal toxicity. Volumetric modulated arc therapy (VMAT) and intensity modulated radiation therapy (IMRT) can also ensure homogenous skin dose if the trade-offs of larger integral doses are warranted. To ensure adequate skin dose coverage, bolus should be considered to bring the full RT dose to the skin surface. Use of bolus can be individualized to the patient's extent of skin disease, the expected skin dose from the plan, and the acute effects experienced during RT. With adequate bolus, all patients should achieve an evident, evolving skin reaction by the first half of treatment.

Standard RT fields and/or contours for IBC should include generous margins on the initially involved skin, especially in the inferior and medial directions. The margin on the medial edge of the mastectomy scar tends to be smaller than the margins seen throughout the rest of the field in order to limit dose to the contralateral breast or chest wall. In fact, in one study of IBC patients treated with post-mastectomy RT, 3 of 5 local failures were at the medial edge of the scar (17). A contouring rule of thumb is to place a minimum $3 \mathrm{~cm}$ margin around the scar as a guide to ensure adequate margins are included in the chest wall target volume. It is also worthwhile to cover the primary chest wall and axillary lymph node drain sites in the primary field with an accompanied $1 \mathrm{~cm}$ margin to ensure they are fully covered. Tumor involvement of the dermal lymphatics and post-operative changes may promote aberrant lymphatic drainage making the failure pattern of IBC somewhat unpredictable. Patients with IBC can have extension of disease via skin and dermal lymphatics from the breast mound to the ipsilateral upper abdomen and axillary midline (Figure $1 A, 1 B$ ). In treatment of IBC, the contours and fields should therefore include the skin of the upper abdomen and lateral breast to at least the mid axillary line. In addition, to allow full margin on the scar at midline, it is reasonable to encroach upon or include a portion of the contralateral breast when needed (Figure 1C). Importantly, the morbidity of local progression to carcinoma en cuirasse in IBC provides a justification for the use of large RT fields.

Because of the propensity of IBC to involve skin, dermal lymphatics, and all regional nodal stations, the standard post-mastectomy RT volumes endorsed by the NCCN guidelines include the chest wall, axillary levels I-III, supraclavicular nodes, and internal mammary nodes (18). A generous chest wall boost is warranted to include all areas of skin initially involved at diagnosis as well as areas where surgical change is evident. Additionally, a 10-15 Gy RT boost to initially involved N3 disease (which are not excised) is required to ensure adequate treatment of this volume. Again, it is critical to review or fuse upfront crosssectional imaging through the neck in $\mathrm{N} 3$ patients and to target all initially gross disease with margins in primary and boost RT fields. The RADCOMP breast atlas designed for a randomized trial incorporating protons is an excellent IBC contouring atlas because it includes the posterior neck nodes and the internal mammary vessel path from the internal mammary nodal spaces superiorly into the subclavicular space (19). These are areas that can be involved in IBC that typically will not get adequate dose without deliberate contouring.

\section{Management of metastatic IBC}

Up to $30 \%$ of patients with IBC present with metastatic disease (1). Historically, patients with metastatic disease have been treated with systemic therapy alone given the dismal survival rates. However, current hypotheses suggest that local therapy, such as surgery or RT, may be beneficial to improve OS in patients with M1 IBC. Recent SEER database analysis reported a 2 -year OS of $39 \%$ in patients with M1 IBC (2). We believe that some M1 disease may represent lymphatic spread without hematogenous spread, and thus may be amenable to aggressive local therapy to achieve durable "no evidence of disease" (NED) status. In addition, unusual presentation of skin metastases can often be treated with individualized RT with the goal of durable NED status. 

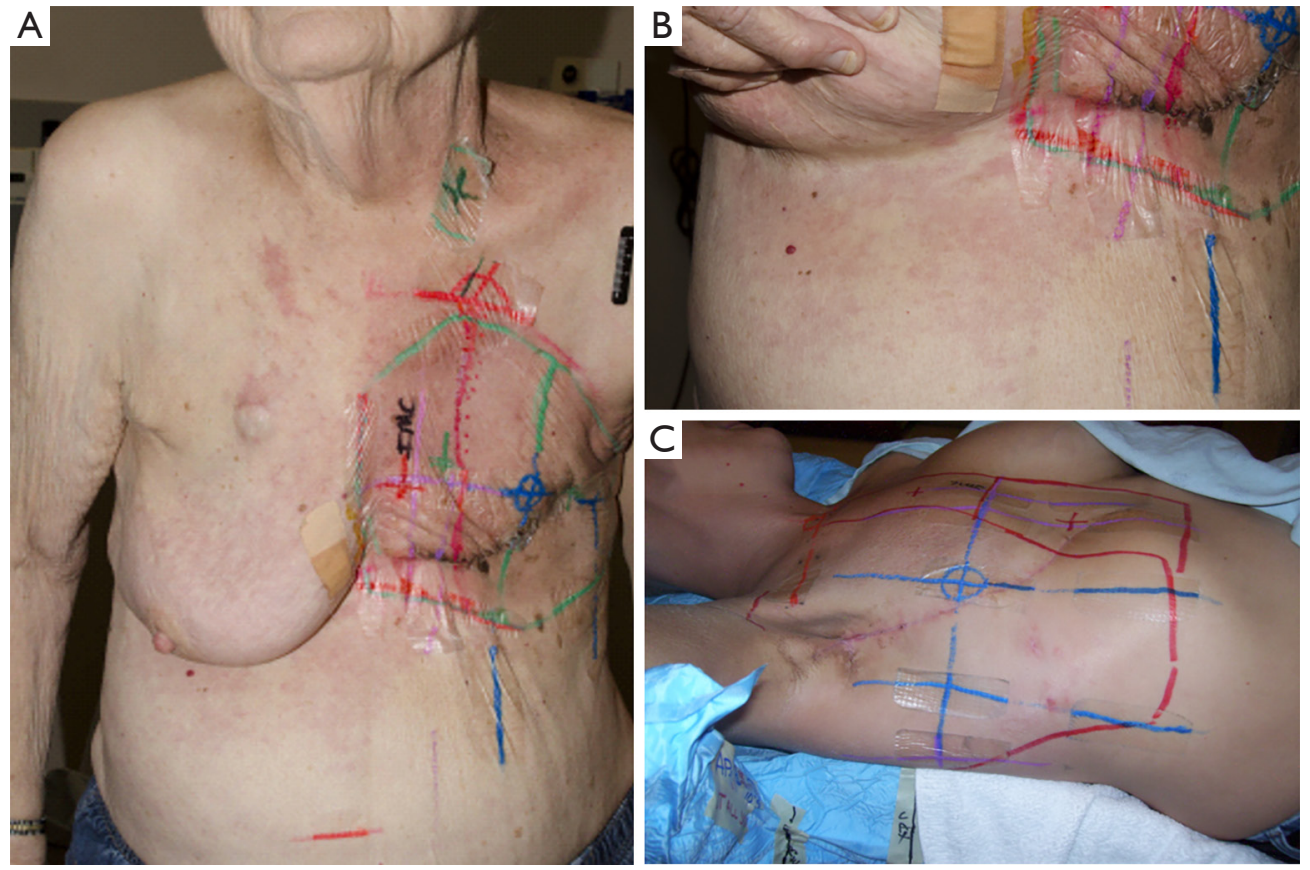

Figure 1 Evidence of progression within the skin of the contralateral abdomen, breast, and infraclavicular area (A). Relationship of inappropriately small RT fields to observed tumor spread into the skin of the abdomen (B). Standard radiation therapy fields for IBC include generous inferior and medial margin which should include the skin of the abdomen and encroach upon or include the contralateral breast (C).

First, it is important to note that IBC may spread to the contralateral nodal basin. In one study, PET-CT imaging data from 177 patients with IBC demonstrated that $27 \%$ of patients had disease in the contralateral nodal basins, and $13(7.3 \%)$ had isolated contralateral disease without other distant metastases. Local control of the contralateral nodal basin(s) was achieved in all 13 patients with either definitive or adjuvant RT (20). Our practice has been to resect these nodes and irradiate the contralateral supraclavicular fossa in these patients. A more recent and larger study evaluated this practice looking at 588 IBC patients independent of imaging modality (21). These authors reported that $8.3 \%$ of IBC patients had synchronous contralateral metastases at the time of diagnosis, and outcomes were not significantly different for those in whom this was the only site of metastatic disease as compared to stage III patients. This suggests that definitive treatment of bilateral disease in patients with known, limited contralateral nodal metastases may have some benefit.

Considering both oligo and polymetastatic presentations, while two randomized trials excluding IBC failed to show a survival benefit to extirpation of the primary breast lesion in metastatic disease, the severe morbidity of local failures in
IBC merits serious consideration of local therapy like that offered to M0 patients. Further, although potentially limited by selection bias, a recent single institution study reported that surgery plus RT was associated with a statistically significant OS benefit compared to either treatment alone in metastatic IBC patients (22). Additionally, local control was four times more likely (HR 0.25 for recurrence) in patients receiving surgery compared to patients who received chemotherapy alone. Similarly, significant differences in local failure (17\% with versus $57 \%$ without local therapy) were observed in a separate single institution study of metastatic IBC patients (23). These findings highlight the value of surgery and RT for local control alone in this population as long as the patient's metastatic disease is controlled, thus allowing for the patient to temporarily stop systemic therapy for the duration of local therapy, and if the patient's life expectancy is long enough to benefit from local control.

\section{Mechanisms of radiation resistance}

Several authors have suggested that radiation resistance is a function of persistent stem-like cancer cells in aggressive 
cancers after radiation exposure (24-26). Van Laere et al. have convincingly demonstrated an enrichment of mammary- or breast cancer-related stem cell signatures in IBC patients following RT receipt (27). Of many purported breast cancer stem cell survival pathways, mevalonate, which is activated in basal breast cancer stem cells, has recently been demonstrated to play an important role in radiation resistance in IBC (28). Specifically, Lacerda et al. demonstrated that simvastatin radiosensitizes mammosphere-initiating cells (MICs) of IBC cell lines but radioprotects MICs of non-IBC cell lines. In a retrospective clinical study of 519 IBC patients treated with post-mastectomy radiation, actuarial 3-year local recurrence-free survival (LRFS) was higher among statin users and, on multivariate analysis, statin use was shown to be independently associated with a higher LRFS (29). These data are intriguing and warrant further investigation particularly considering the findings by Martin and Van Golen demonstrating disparate cholesterol uptake and storage in IBC vs. non-IBC cells (30).

\section{Conclusions}

IBC is an aggressive type of breast cancer that warrants multi-disciplinary care from breast surgical, medical, and radiation oncology. Following neoadjuvant chemotherapy and modified radical mastectomy, adjuvant radiation therapy to the chest wall, as well as to the axillary, internal mammary, infraclavicular, and supraclavicular nodal basins using the RADCOMP atlas contours, is recommended to improve local-regional control rates. Careful RT planning and delivery is necessary to ensure adequate dose and coverage to the at-risk areas of disease, specifically to the upfront skin involvement and any grossly involved regional lymph nodes. Several strategies exist to enhance the effect of RT on local-regional control, including hyperfractionation, use of bolus, increased total RT dose, and radiosensitizers, which are currently being tested in randomized trials. Additionally, generous RT field sizes and contours are justified to decrease the risk of recurrence. With a personalized approach incorporating wide surgical excision of involved skin and dose escalation in higher risk patients, local-regional control rates are improving for IBC.

\section{Acknowledgments}

Funding: This work was supported in part by Cancer Center Support (Core) grant P30 CA016672 from the National
Cancer Institute, National Institutes of Health, to The University of Texas MD Anderson Cancer Center.

\section{Footnote}

Provenance and Peer Review: This article was commissioned by the Guest Editors (Naoto Ueno and Angela Alexander) for the series "Inflammatory Breast Cancer" published in Chinese Clinical Oncology. The article has undergone external peer review.

Reporting Checklist: The authors have completed the Narrative Review reporting checklist. Available at https:// dx.doi.org/10.21037/cco-21-153

Conflicts of Interest: All authors have completed the ICMJE uniform disclosure form (available at https://dx.doi. org/10.21037/cco-21-153). The series "Inflammatory Breast Cancer" was commissioned by the editorial office without any funding or sponsorship. Dr. WAW reports grants from State of Texas, NIH and Komen; consulting fee from Exact Sciences; payment or honoraria from GoToPER; and leadership or fiduciary role in STRO Science Council Vice-Chair and NRG Oncology TS Co-Chair Breast. The authors have no other conflicts of interest to declare.

Ethical Statement: The authors are accountable for all aspects of the work in ensuring that questions related to the accuracy or integrity of any part of the work are appropriately investigated and resolved.

Open Access Statement: This is an Open Access article distributed in accordance with the Creative Commons Attribution-NonCommercial-NoDerivs 4.0 International License (CC BY-NC-ND 4.0), which permits the noncommercial replication and distribution of the article with the strict proviso that no changes or edits are made and the original work is properly cited (including links to both the formal publication through the relevant DOI and the license). See: https://creativecommons.org/licenses/by-nc-nd/4.0/.

\section{References}

1. Wingo PA, Jamison PM, Young JL, et al. Populationbased statistics for women diagnosed with inflammatory breast cancer (United States). Cancer Causes Control 2004;15:321-8.

2. Chang S, Parker SL, Pham T, et al. Inflammatory breast 
carcinoma incidence and survival: the surveillance, epidemiology, and end results program of the National Cancer Institute, 1975-1992. Cancer 1998;82:2366-72.

3. Gonzalez-Angulo AM, Hennessy BT, Broglio K, et al. Trends for inflammatory breast cancer: is survival improving? Oncologist 2007;12:904-12.

4. Walker GV, Niikura N, Yang W, et al. Pretreatment staging positron emission tomography/computed tomography in patients with inflammatory breast cancer influences radiation treatment field designs. Int J Radiat Oncol Biol Phys 2012;83:1381-6.

5. Rueth NM, Lin HY, Bedrosian I, Shaitelman SF, Ueno NT, Shen Y, Babiera G. Underuse of trimodality treatment affects survival for patients with inflammatory breast cancer: an analysis of treatment and survival trends from the National Cancer Database. J Clin Oncol 2014;32:2018-24.

6. Bristol IJ, Woodward WA, Strom EA, et al. Locoregional treatment outcomes after multimodality management of inflammatory breast cancer. Int J Radiat Oncol Biol Phys 2008;72:474-84.

7. Damast S, Ho AY, Montgomery L, et al. Locoregional outcomes of inflammatory breast cancer patients treated with standard fractionation radiation and daily skin bolus in the taxane era. Int J Radiat Oncol Biol Phys 2010;77:1105-12.

8. Rehman S, Reddy CA, Tendulkar RD. Modern outcomes of inflammatory breast cancer. Int J Radiat Oncol Biol Phys 2012;84:619-24.

9. Greenbaum MP, Strom EA, Allen PK, et al. Low locoregional recurrence rates in patients treated after 2000 with doxorubicin based chemotherapy, modified radical mastectomy, and post-mastectomy radiation. Radiother Oncol 2010;95:312-6.

10. Scotti V, Desideri I, Meattini I, et al. Management of inflammatory breast cancer: focus on radiotherapy with an evidence-based approach. Cancer Treat Rev 2013;39:119-24.

11. Ragulin Y, Kurpeshev O, Galkin V, et al. Local hyperthermia for inflammatory breast cancer. Eur J Cancer 2017;72:S11-2.

12. Bristol IJ, Buchholz TA. Inflammatory breast cancer: current concepts in local management. Breast Dis 2005 2006;22:75-83.

13. Liao Z, Strom EA, Buzdar AU, et al. Locoregional irradiation for inflammatory breast cancer: effectiveness of dose escalation in decreasing recurrence. Int J Radiat Oncol Biol Phys 2000;47:1191-200.
14. Stecklein SR, Rosso KJ, Nuanjing J, et al. Excellent Locoregional Control in Inflammatory Breast Cancer With a Personalized Radiation Therapy Approach. Pract Radiat Oncol 2019;9:402-9.

15. Woodward WA. Postmastectomy radiation therapy for inflammatory breast cancer: is more better? Int J Radiat Oncol Biol Phys 2014;89:1004-5.

16. Brown L, Harmsen W, Blanchard M, et al. Once-daily radiation therapy for inflammatory breast cancer. Int J Radiat Oncol Biol Phys 2014;89:997-1003.

17. Takiar V, Akay CL, Stauder MC, et al. Predictors of durable no evidence of disease status in de novo metastatic inflammatory breast cancer patients treated with neoadjuvant chemotherapy and post-mastectomy radiation. Springerplus 2014;3:166.

18. NCCN. Clinical Practice Guidelines in Oncology. Breast cancer, version 8.2021. Accessed November 9, 2021. Available online: https://www.nccn.org/professionals/ physician_gls/pdf/breast.pdf

19. Breast Cancer Atlas. Available online: https://www.rtog. org/CoreLab/ContouringAtlases/BreastCancerAtlas.aspx

20. Woodward WA, Koay E, Takiar V: Radiation therapy for inflammatory breast cancer: technical considerations and diverse clinical scenarios. Breast Cancer Manag 2013;3:43-52.

21. Postlewait LM, Teshome M, Adesoye T, et al. Contralateral Axillary Metastasis in Patients with Inflammatory Breast Cancer. Ann Surg Oncol 2021;28:8610-21.

22. Akay CL, Ueno NT, Chisholm GB, et al. Primary tumor resection as a component of multimodality treatment may improve local control and survival in patients with stage IV inflammatory breast cancer. Cancer 2014;120:1319-28.

23. Warren LE, Guo H, Regan MM, et al. Inflammatory Breast Cancer: Patterns of Failure and the Case for Aggressive Locoregional Management. Ann Surg Oncol 2015;22:2483-91.

24. Chen MS, Woodward WA, Behbod F, et al. Wnt/betacatenin mediates radiation resistance of Sca1+ progenitors in an immortalized mammary gland cell line. J Cell Sci 2007;120:468-77.

25. Phillips TM, McBride WH, Pajonk F. The response of CD24(-/low)/CD44+ breast cancer-initiating cells to radiation. J Natl Cancer Inst 2006;98:1777-85.

26. Woodward WA, Chen MS, Behbod F, et al. WNT/ beta-catenin mediates radiation resistance of mouse mammary progenitor cells. Proc Natl Acad Sci U S A 2007;104:618-23.

27. Van Laere S, Limame R, Van Marck EA, et al. Is there 
Page 8 of 8

a role for mammary stem cells in inflammatory breast carcinoma?: a review of evidence from cell line, animal model, and human tissue sample experiments. Cancer 2010;116:2794-805.

28. Ginestier C, Monville F, Wicinski J, et al. Mevalonate metabolism regulates Basal breast cancer stem cells and is a potential therapeutic target. Stem Cells 2012;30:1327-37.

29. Lacerda L, Reddy JP, Liu D, et al. Simvastatin

Cite this article as: Corrigan KL, Woodward WA, Stauder MC. How should radiation be done for inflammatory breast cancer patients? - a narrative review of modern literature. Chin Clin Oncol 2021;10(6):60. doi: 10.21037/cco-21-153
Corrigan et al. How should radiation be done for IBC patients?

radiosensitizes differentiated and stem-like breast cancer cell lines and is associated with improved local control in inflammatory breast cancer patients treated with postmastectomy radiation. Stem Cells Transl Med 2014;3:849-56.

30. Martin BJ, van Golen KL. A comparison of cholesterol uptake and storage in inflammatory and noninflammatory breast cancer cells. Int J Breast Cancer 2012;2012:412581. 